\title{
Monitoramento Hemodinâmico Invasivo em Pequenos Animais
}

\section{Invasive Hemodynamic Monitoring in Small Animals}

\author{
Márlis Langenegger de Rezende ${ }^{1 *}$; Newton Nunes²; Almir Pereira de Souza ${ }^{1}$; \\ Paulo Sérgio Patto dos Santos ${ }^{1}$
}

\section{Resumo}

Com este artigo objetivou-se revisar os diversos parâmetros hemodinâmicos que podem ser monitorados de maneira invasiva, direta ou indiretamente, como a pressão arterial, pressão venosa central, débito cardíaco, resistência vascular periférica, entre outros. Tais técnicas oferecem uma maior confiabilidade e precisão, além de permitirem um monitoramento contínuo, sendo de grande importância e utilidade no tratamento de pacientes em estado crítico. Por serem técnicas invasivas, não são isentas de risco, cabendo ao profissional, face as vantagens e desvantagens inerentes a cada uma, bem como ao estado clínico do paciente, optar ou não pela sua utilização.

Palavras-chave: monitoramento invasivo, hemodinâmica, pequenos animais

\begin{abstract}
The aim of this paper was to revise the several homodynamic variables that can be monitored by invasive techniques, direct or indirectly, such as arterial pressure, central venous pressure, cardiac output, systemic vascular resistance, among others. Such techniques offer more accuracy and reliability, and also allow a continuous monitoring, being of great importance and utility in the treatment of the critically ill patient. As they are invasive techniques, they aren't free of risks and the professional must decide for their utilization analysing the advantages and disadvantages.
\end{abstract}

Key-words: invasive monitoring, hemodynamics, small animals

\section{Introdução}

A monitoração dos parâmetros fisiológicos vem sendo amplamente empregada durante os procedimentos anestésicos, buscando aumentar sua segurança através da identificação precoce de alterações que possam comprometer a vida do animal. Os pacientes em estado crítico requerem uma monitoração ainda mais próxima, rápida e precisa, objetivando a manutenção e a normalização de suas funções fisiológicas.
As técnicas invasivas oferecem resultados mais confiáveis e precisos, entretanto não são isentas de risco, sendo necessária uma avaliação criteriosa dos riscos e benefícios que possam advir de tais procedimentos.

O monitoramento hemodinâmico inclui parâmetros determinados diretamente como a pressão arterial (PA), pressão venosa central (PVC), débito cardíaco (DC), pressão da artéria pulmonar (PAP) e pressão da artéria pulmonar ocluída (PAPO)

1 Doutorando(a) do Programa de Pós-graduação em Cirurgia Veterinária /FCAV/UNESP/Campus de Jaboticabal-SP.

2 Professor Assistente Doutor, Departamento de Clínica e Cirurgia Veterinária/FCAV/UNESP/Campus de Jaboticabal-SP.

* Autor para correspondência. 
e outros calculados indiretamente através de fórmulas, como a resistência vascular periférica (RVP) e o trabalho do ventrículo esquerdo (TVE).

\section{Pressão arterial (PA)}

A pressão arterial é a pressão sangüínea intraluminal em uma artéria calibrosa. É o produto do débito cardíaco (DC), da resistência vascular periférica (RVP) e do volume sangüíneo (HASKINS, 1999).

A pressão arterial é um indicador da pressão de perfusão tecidual, entretanto, não é o único, e desse modo, não pode ser considerada equivalente ao fluxo sangüíneo nos vários tecidos (NUNES, 2002). A pressão arterial precisa ser significativamente superior à pressão venosa para garantir o fluxo sangüíneo através dos capilares (MANSON; BROWN, 1997). Uma perfusão cerebral e coronariana adequadas requerem uma pressão arterial média mínima de 50 $60 \mathrm{mmHg}$, considerando-se pressão intracraniana normal (HASKINS, 1999). Assim sendo, uma pressão arterial média inferior a $60 \mathrm{mmHg}$ é considerada inaceitável para a manutenção do fluxo sangüíneo tecidual (MANSON; BROWN, 1997).

As drogas anestésicas, os procedimentos cirúrgicos, bem como os eventuais processos fisiopatológicos envolvidos podem causar um comprometimento importante da homeostase cardiovascular, sendo essencial o monitoramento da pressão arterial e através dele a manutenção da mesma em valores satisfatórios (HASKINS, 1996).

A mensuração invasiva da pressão arterial oferece monitoramento contínuo e preciso, mesmo em pacientes com hipotensão grave, sendo portanto mais confiável que a não-invasiva (SAWYER et al., 1991; NUNES et al., 1993). Por outro lado, em se tratando de um método invasivo, faz-se necessário o posicionamento de um cateter intra-arterial, que pode ser realizado através de punção percutânea ou dissecção arterial (Figura 1). A artéria metatársica dorsal e a artéria femoral são as mais utilizadas, sendo a primeira mais empregada para punção percutânea.
Em gatos, a cateterização percutânea é mais facilmente realizada na artéria femoral, embora a artéria metatársica dorsal também possa ser utilizada (TRIM, 1994).

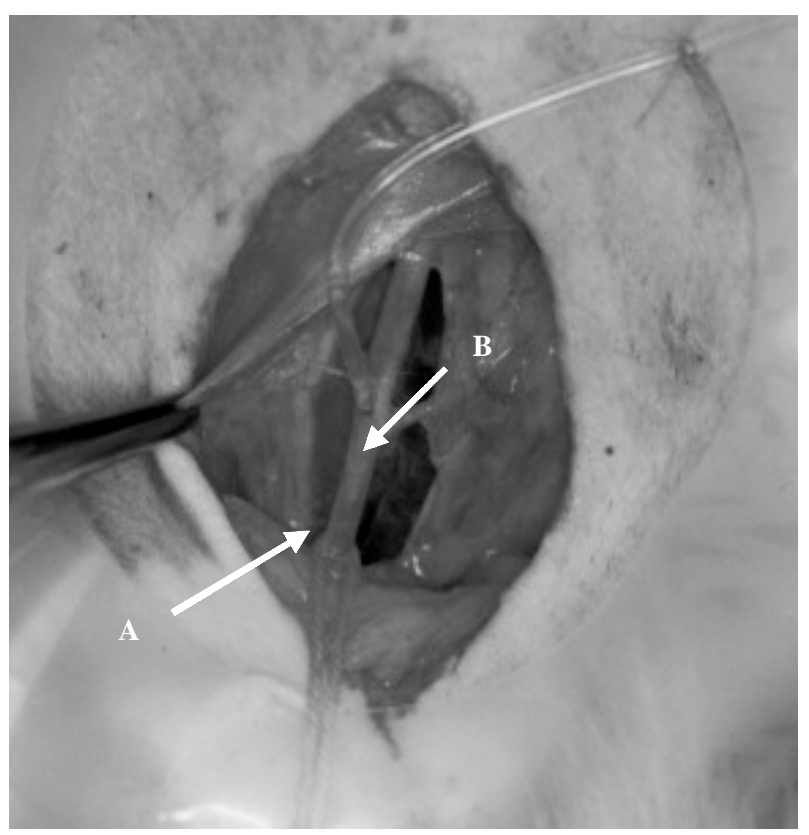

Figura 1 - Dissecção e exteriorização da artéria femoral (A) com introdução de cateter de polietileno (B) intraluminal para mensuração da pressão arterial.

Fonte: Serviço de Anestesiologia em Pequenos Animais do Hospital Veterinário Gov. Laudo Natel - FCAV UNESP - Campus de Jaboticabal.

Imediatamente após o posicionamento do cateter, o mesmo deve ser perfundido freqüentemente com solução salina heparinizada para evitar a coagulação do sangue e trombose (ARAÚJO, 1992; TRIM, 1994; HASKINS,1999; NUNES, 2002). Conecta-se então o cateter ao transdutor elétrico do equipamento de medida através de uma extensão de comprimento variável (preferencialmente não muito longa) e uma torneira de três vias, posicionada entre a extensão e o transdutor.

$\mathrm{O}$ trandutor deve ser posicionado à altura do coração do paciente e deve ser "zerado" (fechando o transdutor para o paciente e abrindo-o para o ar ambiente). Em seguida fecha-se o transdutor para o ar 
ambiente e abre-se a comunicação para o paciente, iniciando-se os registros da PA (ARAÚJO, 1992). Obtém-se um traçado contínuo da onda de pulso, onde a pressão arterial sistólica e diastólica podem ser medidas. Vários monitores apresentam os valores digitais das pressões arteriais sistólica, diastólica e média (TRIM, 1994; NUNES, 2002).

Todo o sistema compreendido entre paciente e transdutor deve estar preenchido com líquido (solução salina heparinizada) e isento de bolhas de ar (NUNES, 2002). A presença de bolhas de ar no sistema provoca uma diminuição na amplitude do pulso, interferindo nos valores de pressão obtidos (BODEY et al., 1994; TRIM, 1994).

O uso do manômetro aneróide é um método básico, simples e barato que permite a mensuração contínua da pressão arterial média (Figura 2). O cateter intra-arterial é conectado à uma extensão que faz a ligação com o manômetro. Uma torneira de três vias é posicionada o mais próximo possível do manômetro, objetivando minimizar a quantidade de ar entre eles. Uma seringa com solução salina heparinizada é conectada à torneira de três vias. Inicialmente abre-se a torneira, de modo que a solução heparinizada flua em direção ao cateter. Após o flush posiciona-se a torneira para que seja estabelecida a ligação entre a artéria e o manômetro (NUNES, 2002). A medida da pressão arterial média se dá pela oscilação do ponteiro entre as pressões sistólica e diastólica (NUNES et al., 1993). Para que a mensuração da pressão seja precisa, a interface entre o ar e a solução salina heparinizada deve estar à altura do coração (TRIM, 1994).

A eventual ligadura da artéria femoral após uma cateterização por dissecção não causa nenhuma complicação circulatória, devido à ampla circulação colateral encontrada nessa região (BURROWS, 1973).

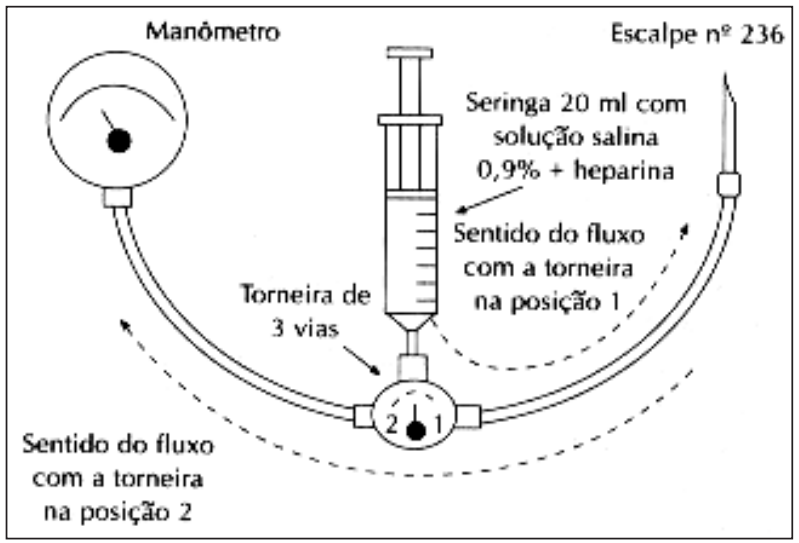

Figura 2 - Representação esquemática do uso do manômetro aneróide para mensuração da pressão arterial. Fonte: Serviço de Anestesiologia em Pequenos Animais do Hospital Veterinário Gov. Laudo Natel - FCAV UNESP - Campus de Jaboticabal.

\section{Pressão venosa central (PVC)}

A pressão venosa central é a pressão sangüínea no lúmen da porção intratorácica da veia cava (HASKINS, 1996) e reflete a pressão no átrio direito (MANSON; BROWN, 1997), proporcionando uma estimativa precisa da pressão de enchimento do ventrículo direito (ARAÚJO, 1992). Os fatores que regulam a PVC são o volume de sangue venoso central (retorno venoso), o tônus vascular venoso e o débito cardíaco (HASKINS, 1999). O grau de complacência do átrio direito durante o preenchimento e a pressão intratorácica também influenciam os valores de PVC (MANSON; BROWN, 1997).

A mensuração da PVC permite estimar a relação entre o volume sangüíneo e a capacidade vascular, bem como a habilidade do coração em bombear o sangue que chega até ele, sendo de grande utilidade nas grandes perdas sangüíneas, quando infusões rápidas de grandes volumes de fluidos são requeridas e quando há suspeita de insuficiência cardíaca direita (MANSON; BROWN, 1997; HASKINS, 1999).

A técnica para mensuração da PVC consiste na introdução de um cateter pela veia jugular, que pode ser feita por punção percutânea ou dissecção venosa, e que deve ser posicionado na porção intratorácica 
da veia cava anterior (Figura 3). O posicionamento no interior do átrio direito deve ser evitado (ARAÚJO, 1992), pois o contato do cateter com endocárdio do átrio ou ventrículo direito pode estimular a atividade de marcapasso ectópico (HASKINS, 1996).

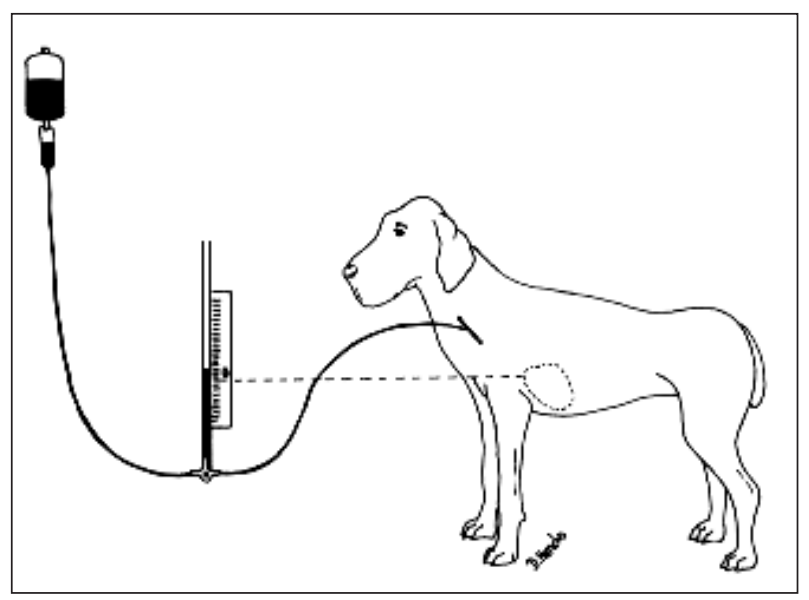

Figura 3 - Representação esquemática do correto posicionamento do cateter para leitura da pressão venosa central na porção intratorácica da veia cava anterior, utilizando a técnica da coluna de água. Observar que o ponto zero da régua fique na altura em que a veia cava atinge o átrio direito.

Fonte: Serviço de Anestesiologia em Pequenos Animais do Hospital Veterinário Gov. Laudo Natel - FCAV UNESP - Campus de Jaboticabal.

O posicionamento correto do cateter é verificado através da oscilação da coluna de água ou da linha de base no monitor eletrônico (ARAÚJO, 1992). Pequenas oscilações devem estar sincronizadas com os batimentos cardíacos e grandes oscilações com os movimentos respiratórios (HASKINS, 1999).

A mensuração da PVC através de coluna de água é um método bastante simples e barato, sendo amplamente utilizado. Primeiramente o animal deve ser posicionado e o ponto zero da régua (que varia de $10 \mathrm{a}+40$ ) ajustado para que fique na altura em que a veia cava atinge o átrio direito. A coluna de água é colocada ao lado da régua de modo que seu volume interno compreenda toda a escala. Na parte inferior da coluna de água acopla-se uma torneira de três vias de modo que uma via seja para o cateter, uma para a própria coluna e a última para um frasco de solução salina a $0,9 \%$. Após o preenchimento do sistema com solução salina, fecha-se esta via, permitindo apenas a ligação entre o cateter e a coluna de água. $\mathrm{O}$ excesso de líquido presente na coluna irá para o paciente e o novo nível atingido representará a PVC (NUNES, 2002). Embora mais acessível, a coluna de água não oferece a mesma precisão que os transdutores eletrônicos mais sofisticados, principalmente quanto à capacidade de resposta às variações de pressão (ARAÚJO, 1992).

A mensuração da PVC deve ser realizada nos intervalos da respiração, pois as alterações da pressão pleural interferem na pressão intraluminal da veia cava (HASKINS, 1999).

\section{Débito cardíaco}

O débito cardíaco é o volume de sangue, em litros, que é bombeado para a circulação sistêmica e pulmonar a cada minuto. Permite avaliar o desempenho da bomba cardíaca, refletindo o volume de sangue disponível para os tecidos (HUG, 1989). Por estar diretamente relacionado à fluxo, fornece indicações muito mais relevantes sobre a perfusão sistêmica, do que os parâmetros pressóricos (HASKINS, 1996).

Existem vários métodos, invasivos e nãoinvasivos, para a mensuração do débito cardíaco, como o método de Fick (consumo de oxigênio), a diluição do corante (cárdio-green), a ecocardiografia e a termodiluição (ARAÚJO, 1992; TRIM, 1994). Entretanto, o método de termodiluição, realizado através do cateter de Swan-Ganz, tem se mostrado o mais confiável (DYSON; ALLEN; MCDONELL., 1985).

Através da técnica de termodiluição, a determinação do débito cardíaco pode ser realizada de forma rápida, segura e repetitiva (ARAÚJO, 1992). Este método requer a utilização do cateter de Swan-Ganz (Figura 4), que é um cateter pulmonar fluxo-dirigido de três vias, que possui um termistor a 5-6 cm da sua extremidade distal e um balonete inflável próximo à sua ponta (ARAÚJO, 1992). O cateter possui 
ainda um conduto que percorre toda a sua extensão e que permite colheitas de valores e curvas de pressão no trajeto e nas cavidades onde é posicionado. Há ainda um segundo conduto que percorre apenas parte de sua extensão e que é a via utilizada para injeção de solução à baixas temperaturas (NUNES, 2002). Existem vários tamanhos de cateter de SwanGanz disponíveis, devendo-se escolher o mais adequado ao porte do paciente. Geralmente utiliza-se o cateter $5 \mathrm{~F}$ para cães de raças pequenas e gatos, e o 7F para cães de raças maiores (NUNES, 2002).

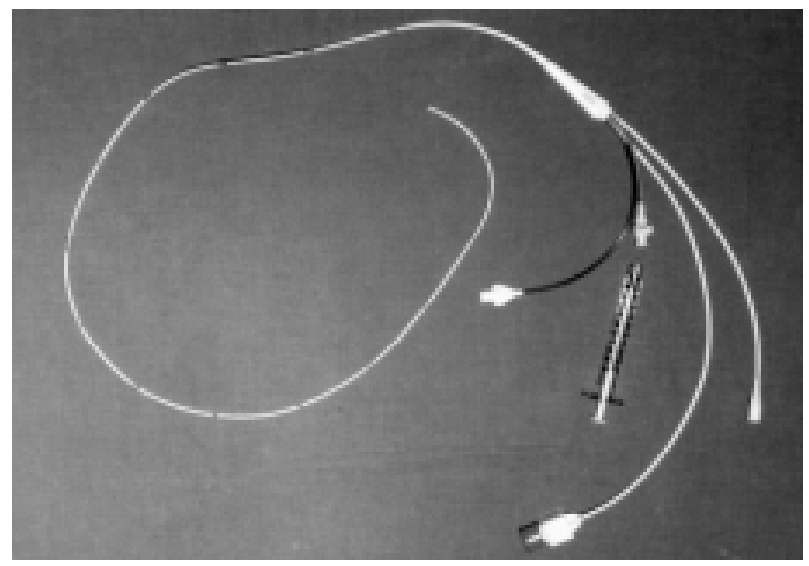

Figura 4 - Cateter de Swan-Ganz, próprio para o monitoramento hemodinâmico invasivo pela técnica da termodiluição.

Fonte: Serviço de Anestesiologia em Pequenos Animais do Hospital Veterinário Gov. Laudo Natel - FCAV UNESP - Campus de Jaboticabal.

A introdução do cateter pode ser feita tanto pela veia jugular (mais freqüente) quanto pela veia femoral, podendo ocorrer por punção percutânea (através de kits de introdução) ou dissecção cirúrgica (NUNES, 2002). O caminho a ser percorrido é constituído pela veia cava, átrio direito, ventrículo direito e chegando por fim à artéria pulmonar (Figura 5) (ARAÚJO, 1992; MASON; BROWN, 1997; NUNES, 2002). O posicionamento correto do cateter é feito através das alterações características das curvas de pressão observadas ao longo de seu trajeto (Figura 6) (GEDDES, 1984; ARAÚJO, 1992; NUNES, 2002).

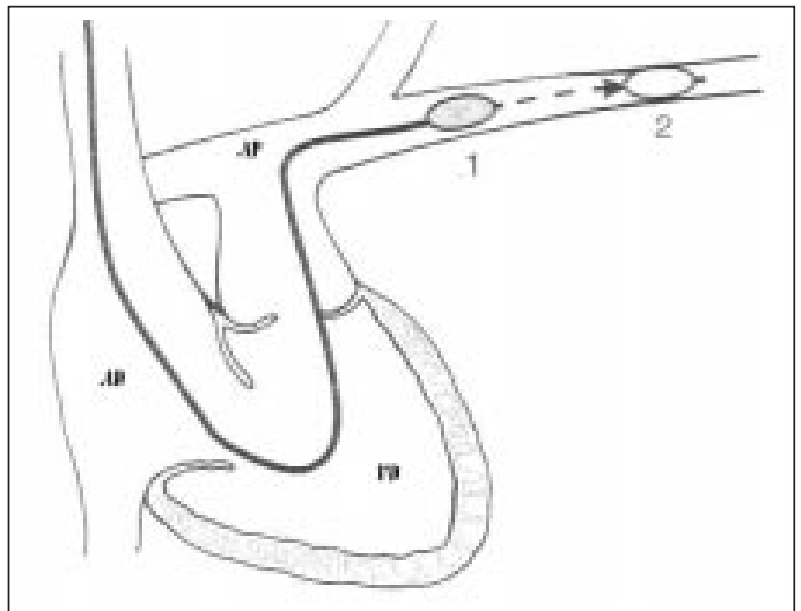

Figura 5 - Representação esquemática do caminho percorrido pelo cateter de Swan-Ganz passando pelo átrio direito (AD), ventrículo direito (VD) e seu correto posicionamento na artéria pulmonar (AP). 1- Cuff do cateter desinflado, AP não ocluída. 2- Cuff do cateter inflado, AP ocluída. Fonte: MARK, J.B. (1998).

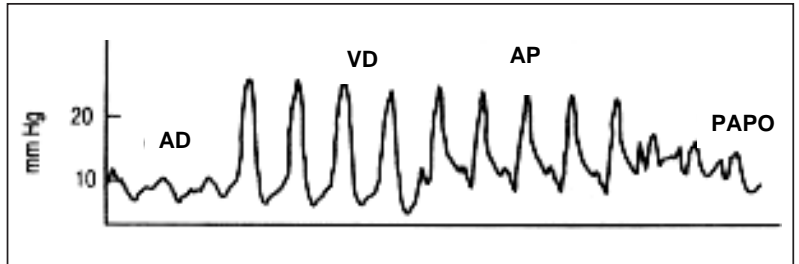

Figura 6 - Representação esquemática dos formatos de onda visualizadas em monitor multiparamétrico, onde podem ser vistas as ondas obtidas no átrio direito (AD), ventrículo direito (VD), artéria pulmonar (AP) e artéria pulmonar ocluída (PAPO).

Fonte: Baxter Healthcare Corporation, Edwards CriticalCare Division. Swan-Ganz Thermodilution Catheters Illustrated Manual.

A técnica de mensuração do débito cardíaco baseia-se na diferença de temperatura entre o líquido injetado e a temperatura corpórea central do paciente. Informa-se ao computador de débito cardíaco o volume a ser injetado e a temperatura do mesmo (geralmente $0^{\circ} \mathrm{C}$ ), e o termistor na ponta do cateter de Sawn-Ganz informa a temperatura do paciente. $\mathrm{O}$ computador mede a mudança de temperatura que ocorre durante o tempo na artéria pulmonar imediatamente após a injeção de solução gelada no átrio ou cava (ARAÚJO, 1992). A curva de termodiluição obtida é visualizada na tela e o computador calcula a área sob a curva, que corresponde ao débito cardía- 
co, e fornece o valor em 1/min (ARAÚJO, 1992; NUNES, 2002). Devem ser realizadas de três a cinco medidas, das quais se obtém uma média que representará um valor mais fidedigno de débito cardíaco (NUNES, 2002). Para que os valores obtidos sejam ainda mais precisos e confiáveis, maior deve ser a diferença entre a temperatura da solução injetada e a temperatura corpórea central do paciente, sendo por isso geralmente utilizada a solução a $0^{\circ} \mathrm{C}$ (ARAÚJO, 1992). Vale ressaltar que animais de portes diferentes, produzirão valores de débito cardíaco diferentes, sendo interessante calcular o índice cardíaco (débito cardíaco/peso corpóreo) para que se possam obter valores comparáveis entre os diversos pacientes (NUNES, 2002).

O cateter de Swan-Ganz permite ainda a monitoração direta da pressão arterial pulmonar (PAP); da pressão da artéria pulmonar ocluída (PAPO), também chamada pressão capilar pulmonar e que reflete a pressão atrial esquerda; da pressão venosa central (PVC) ou atrial; além da colheita de sangue venoso misto (na artéria pulmonar, com o balão desinflado) e sangue venoso (na veia cava ou átrio direito) (ARAÚJO, 1992; MASON; BROWN, 1997).

Outros parâmetros podem ser calculados através de fórmulas como (a) volume sistólico (VS) e índice sistólico (IS): VS = DC/FC e IS = VS/ASC (MUIR; MASON, 1996), onde: DC= Débito Cardíaco (ml/ $\min$.), $\mathrm{FC}=$ Freqüência Cardíaca (batimentos/min.), $\mathrm{VS}=$ Volume Sistólico (ml/batimento), ASC= Área da Superf. Corpórea $\left(\mathrm{m}^{2}\right)$ e IS= Índice Sistólico (ml/ batimento $\mathrm{x} \mathrm{m}^{2}$ ); (b) trabalho ventricular esquerdo (TVE) e índice do trabalho ventricular esquerdo (ITVE): TVE = PAM $\times$ DC $\times 0,0135$ e ITVE = TVE/ASC (VALVERDE, 1991), onde: 0,0135 = Fator de Correção ( $\mathrm{L}$ mmHg para $\mathrm{Kg}$ x m), PAM= Pressão Arterial Média ( $\mathrm{mmHg}$ ), DC = Débito Cardíaco (L/min.), ASC = Área da Superf. Corpórea $\left(\mathrm{m}^{2}\right)$, TVE $=$ Trabalho Ventricular Esquerdo ( $\mathrm{Kg} \times \mathrm{m} / \mathrm{min}$.), ITVE= Índice do TVE (Kg x m/ min. $\left.\mathrm{x} \mathrm{m}^{2}\right)$; (c) resistência periférica total (RPT) e índice da resistência periférica total $($ IRPT $): \mathrm{RPT}=(\mathrm{PAM} / \mathrm{DC}) \mathrm{x}$ 79.9 e IRPT $=$ RPT $\times$ ASC, onde: $79,9=$ Fator de Correção $\left(\mathrm{mmHg} x \mathrm{~min} . / \mathrm{L}\right.$ para dina $\left.\mathrm{x} \operatorname{seg} / \mathrm{cm}^{5}\right)$,

PAM = Pressão Arterial Média $(\mathrm{mmHg}), \mathrm{DC}=$ Débito Cardíaco (L/min.), ASC = Área da Superf. Corpórea $\left(\mathrm{m}^{2}\right), \mathrm{RTP}=$ Resistência Periférica Total (dina $x$ seg. $\left./ \mathrm{cm}^{5}\right)$ e IRPT $=$ Índice da RPT (dina $\mathrm{x}$ seg. / $\mathrm{cm}^{5} \mathrm{x} \mathrm{m}^{2}$ ) (VALVERDE, 1991); (d) resistência vascular pulmonar (RVP) e índice da resistência vascular pulmonar $(\mathrm{IRVP}): \mathrm{RVP}=[(\mathrm{PAPm}-\mathrm{PCPm})$ / DC] $\times 79.92$ e IRVP = (RVP x ASC), onde: $\mathrm{PAPm}=$ Pressão Art. Pulmonar Média (mmHg), PCPm= Pressão Capilar Pulmonar Média (mmHg), DC= Débito Cardíaco (L/min.), ASC= Área da Superf. Corpórea $\left(\mathrm{m}^{2}\right), 79,9=$ Fator de Correção (mmHg x min./L para dina $\mathrm{x}$ seg $\left./ \mathrm{cm}^{5}\right), \mathrm{RVP}=$ Resistência Vascular Pulmonar (dina $x$ seg. $/ \mathrm{cm}^{5}$ ) e IRVP= Ïndice da RVP (dina $\mathrm{x}$ seg. $/ \mathrm{cm}^{5} \mathrm{x} \mathrm{m}^{2}$ ); (e) pressão de perfusão coronariana $(\mathrm{PPC}): \mathrm{PPC}=\mathrm{PAM}-\mathrm{PADm}$, onde: $\mathrm{PAM}=$ Pressão Arterial Média $(\mathrm{mmHg}), \mathrm{PADm}=$ Pressão Média do Átrio Direito $(\mathrm{mmHg}), \mathrm{PPC}=$ Pressão de Perfusão Coronariana $(\mathrm{mmHg}$ ) (ARAÚJO, 1992; MASON; BROWN, 1997; NUNES, 2002).

Assim sendo, apenas com a cateterização do lado direito da circulação sangüínea é possível obter o perfil hemodinâmico do paciente em estado crítico (ARAÚJO, 1992). Os valores normais de pressão intracardíaca e intravascular em pequenos animais podem ser consultados na Tabela 1 .

Tabela 1 - Pressões intravasculares e intracardíacas normais $(\mathrm{mmHg})$ em pequenos animais*

\begin{tabular}{lccc}
\hline Local & Sistólica & Diastólica & Média \\
\hline Átrio direito (PVC) & $4-6$ & $0-4$ & $2-5$ \\
Ventrículo direito & $15-30$ & $<5$ & -- \\
Arterial sistêmica & $110-160$ & $80-110$ & $90-120$ \\
Átrio esquerdo & $5-12$ & $<8$ & $<10$ \\
Ventrículo esquerdo & $95-150$ & $<10$ & -- \\
Aorta & $95-150$ & $70-100$ & $80-110$ \\
Artéria pulmonar & $15-30$ & $5-15$ & $8-20$ \\
Artéria pulmonar ocluída & $6-12$ & $4-8$ & $5-10$ \\
& & \\
*Os valores aqui apresentados são os esperados em cães e gatos \\
submetidos à anestesia geral. Fonte: Modificado de KIENLE(1998).
\end{tabular}




\section{Considerações finais}

O monitoramento hemodinâmico invasivo representa uma grande evolução no tratamento de pacientes em estado crítico, pois oferece um acompanhamento muito mais próximo, detalhado e fidedigno das condições cardiovasculares desses pacientes, permitindo assim que o Médico Veterinário atue de forma precoce, precisa e eficiente, no controle, manutenção e restauração de suas funções fisiológicas.

\section{Referências}

ARAÚJO, S. Monitorização hemodinâmica invasiva à beira do leito. In: TERZI, R. Técnicas básicas em UTI. São Paulo: Manole, 1992. p.143-181.

BODEY, A.R. et al. A comparison of direct and indirect (oscilometric) measurement of arterial blood pressure in anesthetized dogs using tail and limbs cuffs. Research and Veterinary Science, London, v.57, p.265-269, 1994.

BURROWS, C.F. Techniques and complications of intraveous and intraarterial catheterization in dogs and cats. Journal of American Veterinary Medicine Association, v.163, p.1357-1363, 1973.

DYSON, D.H.; ALLEN, D. G.; McDONELL, W. N. Comparison of three methods for cardiac output determination in cats. American Journal of Veterinary Research, Chicago, v.46, p.2546-2552, 1985.

HASKINS, S.C. Monitoring the anesthetized patient. In: THURMON, J.C.; TRANQUILLI, W.J.; BENSON, G.J. Lumb \&Jones' Veterinary Anesthesia. 3.ed. Baltimore: Williams \& Wilkins, 1996. p. 409-423.

HASKINS, S.C. Perioperative monitoring. In: PADDLEFORD, R.R. Manual of small animal anesthesia. 2.ed. Philadelphia: W.B. Sauders, 1999. p.123-146.
HUG, C.J. Monitoramento. In: MILLER, R.D. Tratado de anestesia. 2.ed. São Paulo: Manole, 1989. p.419-471.

KIENLE, R. D. Cardiac catheterization. In: KITTLESON, M. D. Small animal cardiovascular medicine. St. Louis: Mosby Inc., 1998. Chapter 7. p.118-132.

MARK, J. B. Predicting left ventricular and - diastolic pressure. In:_____. Atlas of cardiovascular monitoring. New York: Churchill Livingstone. 1998. Chapter 6, p.60-79.

MASON, D.E.; BROWN, M.J. Monitoring of anesthesia. In: KOHN, D.F et al. Anesthesia and analgesia in laboratory animals. London: Academic Press, 1997. p.73-81.

MUIR, W.W.; MASON, D. Cardiovascular System. In: THURMON, J.C., TRANQUILLI, W.J.; BENSON, G.J. Lumb \& Jones' veterinary anesthesia. 3.ed. Philadelphia: Lea \& Feabiger, 1996. p.80.

NUNES, N.; COSTA, J.L.O.; NÓBREGA NETO, P.I. Efeitos cardiorrespiratórios da anestesia epidural em cães não pré-medicados. Ciência Rural, Santa Maria, v.23, n.3, p.305-309, 1993.

NUNES, N. Monitoração da anestesia. In: FANTONI, D.T.; CORTOPASSI, S.R.G. Anestesia em cães e gatos. São Paulo: Rocca, 2002. p.64-81.

SAWYER, D.C. et al. Comparison of direct and indirect blood pressure in anesthetized dogs. Laboratory Animal Science, Cordova, v.41, n.2, p.134-138, 1991.

TRIM, C.M. Monitoring the anaesthetized cat. In: HALL, L.W.; TAYLOR, P.M. Anaesthesia of the cat. London: Baillière Tindal, 1994. p.194-223.

VALVERDE, A. Comparison of the hemodynamic effects of halothane alone and halothane combined with epidurally administered morphine for anesthesia in ventilated dogs. American Journal of Veterinary Research, Chicago, v.52, n.3, p.505-509, 1991. 
